\title{
A novel path sampling method for the calculation of rate constants
}

\author{
Titus S. van Erp, Daniele Moroni, and Peter G. Bolhuis \\ Department of Chemical Engineering, Universiteit van Amsterdam, Nieuwe Achtergracht 166, \\ 1018 WV Amsterdam, The Netherlands
}

(Received 5 November 2002; accepted 30 January 2003)

\begin{abstract}
We derive a novel efficient scheme to measure the rate constant of transitions between stable states separated by high free energy barriers in a complex environment within the framework of transition path sampling. The method is based on directly and simultaneously measuring the fluxes through many phase space interfaces and increases the efficiency with at least a factor of 2 with respect to existing transition path sampling rate constant algorithms. The new algorithm is illustrated on the isomerization of a diatomic molecule immersed in a simple fluid. (C) 2003 American Institute of Physics. [DOI: 10.1063/1.1562614]
\end{abstract}

\section{INTRODUCTION}

The calculation of rate constants of activated processes dominated by rare events, chemical reactions being a prime example, is still one of the major computational challenges. As transition rates depend exponentially on the activation barrier height, the expectation time for an event can exceed current computer capabilities by many orders of magnitude. As a result most chemical reactions cannot be simulated by direct molecular dynamics (MD) methods, except those with very low activation energies. The conventional way to tackle this time scale problem is based on transition state theory (TST) and separates the problem in two steps. ${ }^{1-4}$ The first step is the calculation of the free energy barrier as function of a reaction coordinate, the second stage is the calculation of the transmission coefficient by sampling fleeting trajectories departing from the top of the barrier. If the reaction coordinate is well chosen, the top of the free energy barrier corresponds to points in phase space close to the true transition state, and the transmission coefficient will have a reasonable value. However, in high dimensional complex systems the choice of reaction coordinate can be extremely difficult and usually requires detailed a priori knowledge of the transition mechanism. Consequently, an intuitively chosen but wrong reaction coordinate can result in a very low transmission coefficient, and hence a statistically inaccurate or immeasurable rate constant.

Chandler and co-workers ${ }^{5-9}$ devised a method for which no prior knowledge of the system is needed. This method, called transition path sampling (TPS), gathers a collection of trajectories connecting the reactant to the product region by employing a Monte Carlo (MC) algorithm. The resulting path ensemble can be used to elucidate reaction mechanisms, transition states, and reaction coordinates. The TPS method has been successfully used on such diverse systems as cluster isomerization, autodissociation of water, ion pair dissociation and on isomerization of a dipeptide, as well a reactions in aqueous solution (see Ref. 8 for an overview). Just as in the conventional case mentioned above, an additional second simulation is needed to determine the rate constant within TPS. This simulation combines the path sampling method with the umbrella sampling technique to estimate the probability to reach the product state from the initial reactant state. The final macroscopic rate constant is given by a plateau in the time derivative of a correlation function. ${ }^{7}$ In case of two distinct stable states this plateau region should always exist at times longer than the typical molecular relaxation time. However, when reaction pathways are complex and exhibit multiple recrossings, these typical molecular relaxation timescales can be relatively long. In that case the TPS rate constant calculation is computationally expensive, as the path length must exceed these time scales.

In this paper we improve the efficiency of the TPS rate constant calculation on several points by introducing an alternative scheme for calculating reaction rates, named transition interface sampling (TIS). The first of these improvements is allowing the path length to vary, so that by a well chosen definition of the stable states we can limit the length of each path to the strict minimum. Second, the new method is based on the effective positive flux through dividing surfaces or interfaces and is consequently much less sensitive to multiple recrossings or diffusive barrier crossings. Third, the number of different types of Monte Carlo moves is reduced, making the implementation of the algorithm conceptually simpler.

This paper is organized as follows: In Sec. II we briefly describe the existing algorithms and present the theoretical derivation for the TIS rate constant expression. The implementation of the algorithm is discussed in Sec. III. We illustrate the algorithm on a diatomic molecule in a fluid of repulsive particles and make a quantitative comparison to the original TPS calculation in Sec. IV. We end with concluding remarks in Sec. V.

\section{THEORY}

\section{A. Transition state theory and the calculation of rate constants}

Consider a dynamical system in which transitions can take place between two stable states $A$ and $B$. If the barrier between $A$ and $B$ is sufficiently high, the system will show exponential relaxation for which the forward and backward 
rate constants $k_{A B}$ and $k_{B A}$ are well defined and can be expressed in terms of microscopic properties. Measuring these rate constants by computer simulation is traditionally done by the two stage Bennett-Chandler (BC) procedure based on the principles of TST. ${ }^{3,4}$ The first step is the calculation of the reversible work or free energy to bring the system from stable state $A$ to the transition state. This free energy $F(\lambda)$ has to be calculated as a function of a suitably chosen reaction coordinate $\lambda$. This $\lambda$ can be a complex function of all particle coordinates $r$ and momenta $p: \lambda=\lambda(x)$, with $x$ $\equiv\{r, p\}$. The maximum in $F(\lambda)$ defines the transition state dividing surface $\lambda^{*}{ }^{10,11}$ By convention, the system is in $A$ if $\lambda(x)<\lambda^{*}$ and in $B$ if $\lambda(x)>\lambda^{*}$.

The main assumption in TST is that any trajectory coming from $A$ and crossing the transition state dividing surface $\lambda(x)=\lambda^{*}$ will remain at the $B$ side of the dividing surface for a long time. The reaction rate can therefore be expressed as the positive flux through the multidimensional dividing surface $\lambda^{*}$,

$$
\begin{aligned}
k_{A B}^{\mathrm{TST}} & =\lim _{\Delta t \rightarrow 0} \frac{1}{\Delta t} \frac{\left\langle\theta\left(\lambda^{*}-\lambda\left(x_{0}\right)\right) \theta\left(\lambda\left(x_{\Delta t}\right)-\lambda^{*}\right)\right\rangle}{\left\langle\theta\left(\lambda^{*}-\lambda\right)\right\rangle} \\
& =\frac{\left\langle\dot{\lambda}\left(x_{0}\right) \delta\left(\lambda\left(x_{0}\right)-\lambda^{*}\right) \theta\left(\dot{\lambda}\left(x_{0}\right)\right)\right\rangle}{\left\langle\theta\left(\lambda^{*}-\lambda\right)\right\rangle} \\
& =\left\langle\dot{\lambda}\left(x_{0}\right) \theta\left(\dot{\lambda}\left(x_{0}\right)\right)\right\rangle_{\lambda *} \frac{e^{-\beta F\left(\lambda^{*}\right)}}{\int_{-\infty}^{\lambda^{*}} e^{-\beta F(\lambda)} d \lambda},
\end{aligned}
$$

where $x_{t}$ specifies the set of coordinates and momenta of the system at time $t$, the dots denote derivatives with respect to time $t$, the brackets $\langle\cdots\rangle$ denote equilibrium ensemble averages, and $\theta(x)$ and $\delta(x)$ are the Heaviside step-function and the Dirac delta function, respectively. In the last equality of Eq. (1) the connection to the reversible work $F(\lambda)$ is made, and $\beta=1 / k_{B} T$, where $k_{B}$ is Boltzmann's constant and $T$ is the temperature. The subscript $\lambda *$ to the ensemble brackets, indicates that the ensemble is constrained to the top of the barrier $\lambda^{*}$

We consider the system to be completely deterministic and thus we can write $x_{t}=f\left(x_{t^{\prime}}, t-t^{\prime}\right)=f\left(x_{0}, t\right)$, in which $f$ is the time-propagator function. Evaluation of the function $f(x, t)$ requires integrating the equations of motion over the time interval $t$ starting with configuration $x$. Nevertheless, the equations derived in this paper are still valid when applied to stochastic dynamics.

Even when the TST assumption is accurate, it can be extremely difficult to find a proper reaction coordinate for which recrossings do not occur. As a result a wrong choice for the reaction coordinate will give a much lower free energy barrier than the real activation free energy and will correspondingly overestimate the rate constant. Figure 1 illustrates that Eq. (1) overcounts trajectories. One can correct for this overcounting by multiplying the TST rate constant with the transmission coefficient $\kappa(t)$ to obtain the true rate constant

$$
k_{A B}(t)=k_{A B}^{\mathrm{TST}} \kappa(t) .
$$

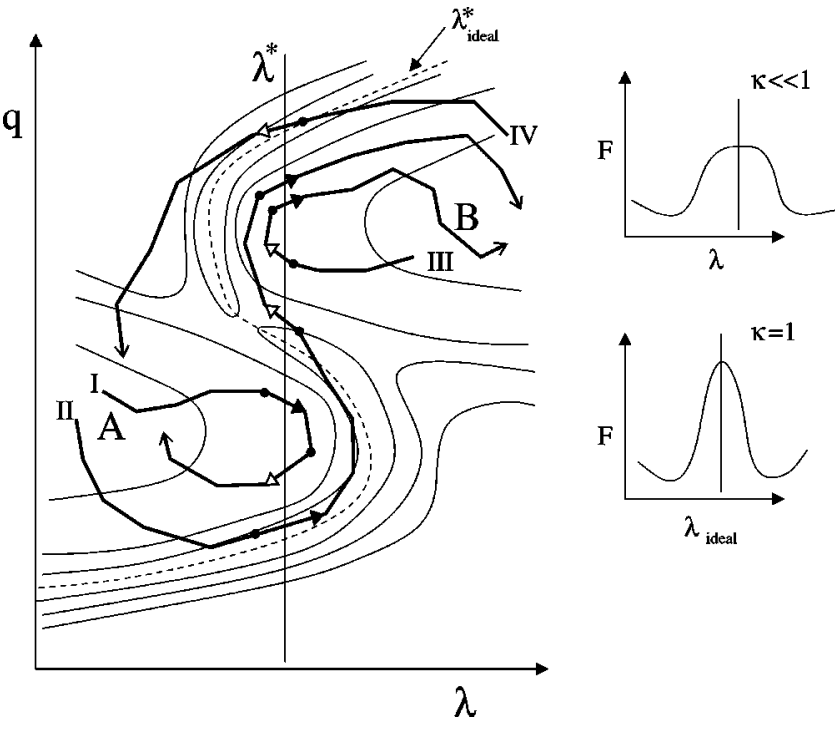

FIG. 1. The thin solid curves show the two-dimensional free energy landscape in the contour plot. $\lambda$ is the chosen reaction coordinate, $q$ represents all other degrees of freedom. $A$ and $B$ denote the state regions. The vertical line at $\lambda *$ corresponds to the maximum in the free energy function $F(\lambda)$ as is shown at the right upper side. The free energy as function of the ideal reaction coordinate is also shown at the right lower side. This reaction coordinate is a complex function of all degrees of freedom $\lambda_{\text {ideal }}$ $=\lambda_{\text {ideal }}(\lambda, q)$ and the corresponding free energy function has its maximum at the true transition state dividing surface $\lambda_{\text {ideal }}=\lambda_{\text {ideal }}^{*}$. This true dividing surface is the dashed curved line. The corresponding free energy barrier is much more narrow and higher than the artificial barrier due to the incorrect reaction coordinate. Four possible trajectories are shown. The black solid arrows indicate a positive flux through the surface $\lambda^{*}$ and the white solid arrows indicate the negative fluxes. TST rate expression (1) counts all positive fluxes of trajectories I, II, and III. Consequently, nontrue reactive events like I and III have an artificial contribution to the rate constant and also trajectory II is overcounted one time. To correct for this, one can calculate the transmission coefficient $\kappa$. In the TPS equation (4), if $\lambda_{A}=\lambda_{B}=\lambda^{*}$, trajectories III and IV are not counted because of the $h_{A}\left(x_{0}\right)$ term. Trajectories I and II are correctly counted in the final summation due to the cancellation of positive and negative flux terms.

The calculation of the time dependent transmission coefficient $\kappa(t)$ constitutes the second part of the two stage BC procedure. ${ }^{3,4} \kappa(t)$ belongs to the approximate dividing surface $\lambda^{*},{ }^{1-4}$ and can be determined by taking an ensemble average of many short trajectories starting from the dividing surface,

$$
\kappa(t)=\frac{1}{\left\langle\dot{\lambda}\left(x_{0}\right) \theta\left(\dot{\lambda}\left(x_{0}\right)\right)\right\rangle_{\lambda *}}\left\langle\dot{\lambda}\left(x_{0}\right) \theta\left(\lambda\left(x_{t}\right)-\lambda^{*}\right)\right\rangle_{\lambda^{*}} .
$$

After a short molecular time $t_{\text {mol }}$ the trajectories are committed to a stable state and $\kappa(t)$, and hence $k_{A B}(t)$, become constant: the transmission coefficient $\kappa$, and the rate constant $k_{A B}$, respectively. It is however important to start sufficiently close to the true transition state dividing surface. Otherwise the transmission coefficient will be extremely low, making an accurate estimate of the rate constant problematic or even impossible. In many cases, in particular for complex condensed matter systems, a sufficiently close reaction coordinate is difficult to find and requires considerable a priori knowledge about the system. 


\section{B. Transition path sampling}

Transition path sampling (TPS) is developed to overcome the difficulties mentioned above. ${ }^{5-9}$ Its main advantage is that no prior knowledge of the transition state is needed. The rate constant in TPS is expressed as the time derivative of a general time correlation function.

$$
k_{A B}^{\mathrm{TPS}}(t)=\frac{\mathrm{d}}{\mathrm{d} t} C(t), \quad C(t)=\frac{\left\langle h_{A}\left(x_{0}\right) h_{B}\left(x_{t}\right)\right\rangle}{\left\langle h_{A}\left(x_{0}\right)\right\rangle},
$$

in which $h_{A}(x)$ and $h_{B}(x)$ are the characteristic functions defined by

$$
\begin{array}{llll}
h_{A}(x)=1, & \text { if } x \in A, & \text { else } & h_{A}(x)=0, \\
h_{B}(x)=1, & \text { if } x \in B, & \text { else } & h_{B}(x)=0 .
\end{array}
$$

In case of a single order parameter $\lambda\left(x_{t}\right)$ describing the transition, the phase space regions $A$ and $B$ are defined by $\lambda_{A}$ and $\lambda_{B}: x_{t} \in A$ if $\lambda\left(x_{t}\right)<\lambda_{A}$ and $x_{t} \in B$ if $\lambda\left(x_{t}\right)>\lambda_{B}$. Knowledge of the precise location of the dividing surface $\lambda^{*}, \lambda_{A}<\lambda^{*}<\lambda_{B}$, is not required in TPS. Therefore, the order parameter $\lambda$ does usually not correspond to the reaction coordinate.

The microscopic expression for the rate constant in Eq. (4) is time dependent, while the phenomenological rate constant is not. However, just as the transmission coefficient $\kappa(t)$ becomes a constant, the time dependent function $k_{A B}^{\mathrm{TPS}}(t)$ reaches a plateau after a molecular time scale $t_{\mathrm{mol}}$. The phenomenological rate constant is equal to the plateau value, $k_{A B}=k_{A B}^{\mathrm{TPS}}(T)$. This plateau region should always exist for times $T$ between the molecular timescale and the characteristic reaction time: $t_{\mathrm{mol}}<T \ll t_{\mathrm{rxn}}$. In other words, $T$ is larger than the time scale to commit to one of the stable states, but much shorter than the expectation time $t_{\mathrm{rxn}}$ of a completely new reactive event. If we take $\lambda_{A}=\lambda_{B}=\lambda^{*}$ and the limit $t \rightarrow 0+$, Eq. (4) transforms into the expression for the positive reactive flux or, equivalently, the TST equation (1). For $t>0$, however, the reactive flux measured by Eq. (4) no longer consists of purely positive contributions. The final rate constant is a sum of positive and negative fluxes, and thus the overcounting of trajectories in Eq. (1) is circumvented (see Fig. 1).

We can rewrite the time dependent rate constant of Eq. (4) into $^{7}$

$$
k_{A B}^{\mathrm{TPS}}(t)=\frac{\left\langle\dot{h}_{B}(t)\right\rangle_{A, H_{B}(T)}}{\left\langle h_{B}\left(t^{\prime}\right)\right\rangle_{A, H_{B}(T)}} \cdot C\left(t^{\prime}\right),
$$

where $H_{B}(T)=\max _{0<t<T} h_{B}\left(x_{t}\right)$ and $\langle\cdots\rangle_{A, H_{B}(T)}$ denotes an average on the ensemble of paths of fixed length $T$ starting in $A$ and entering $B$ at least once. ${ }^{7}$ These ensemble averages are evaluated using a Monte Carlo procedure employing the shooting and shifting moves. ${ }^{6}$ The two factors in Eq. (6) have to be evaluated separately. First, a path sampling simulation is performed to compute $\left\langle h_{B}(t)\right\rangle_{A, H_{B}(T)}$ in the interval $[0, T]$. The path length $T$ must be long enough for the time derivative to display a plateau. Subsequently, one chooses a $t^{\prime}$ in interval $[0, T]$ and computes $C\left(t^{\prime}\right)$ using the path sampling in combination with an umbrella sampling technique. ${ }^{7} \mathrm{~A}$ drawback of the TPS rate constant calculation is that the function $k_{A B}^{\mathrm{TPS}}(t)$ can be strongly oscillatory because of recrossings and will reach a plateau only after a relatively long time. The path length in TPS must exceed the typical timescale of these oscillations, and consequently, in that case TPS is computationally costly.

\section{Transition interface sampling}

Just as the BC and the TPS rate constant algorithms, the TIS method is based on a flux calculation. In contrast to these schemes, however, TIS measures the effective positive flux. ${ }^{12}$ instead of a conditional general flux as in Eq. (4) or Eq. (3). This implies that only positive terms contribute to the rate, allowing for faster numerical convergence. A flux is normally defined through a hypersurface in phase space defined by an order parameter, the reaction coordinate. But, similar to the TPS case, we do not want to suffer from a bad choice of reaction coordinate. Therefore, instead of using a single dividing surface, we introduce a series of interfaces through which we measure this flux. We then derive an expression that relates the flux through a certain interface to the flux through an interface which is closer to $A$ to replace the expensive TPS umbrella sampling procedure.

In order to formulate a proper flux, we have to divide the entire phase space into two complementary regions called overall states $\mathcal{A}$ and $\mathcal{B}$. These states do not only depend on the position at the time of consideration but also on its past behavior. Overall state $\mathcal{A}$ covers all phase space points lying inside stable region $A$, which constitutes the largest part, but also all phase space points that visit $A$, before reaching $B$ when the equations of motion are integrated backward in time. Similarly, state $\mathcal{B}$ comprises stable state $B$ and all phase points, coming directly from this state in the past, i.e. without having been in $A$. It is useful to generalize the characteristic functions in Eq. (5) for an arbitrary phase space region $\Omega$,

$$
h_{\Omega}(x)=1, \quad \text { if } x \in \Omega, \text { else } h_{\Omega}(x)=0 .
$$

For each phase point $x$ and each phase space region $\Omega$ we can determine the minimum (first entrance) times $t_{\Omega}^{b}(x)$ and $t_{\Omega}^{f}(x)$ needed to reach $\Omega$ starting from configuration $x$ by integrating the equations of motion backward and forward in time, respectively,

$$
\begin{aligned}
& t_{\Omega}^{b}(x) \equiv-\max \left[\left\{t \mid h_{\Omega}(f(x, t))=1 \wedge t \leqslant 0\right\}\right], \\
& t_{\Omega}^{f}(x) \equiv+\min \left[\left\{t \mid h_{\Omega}(f(x, t))=1 \wedge t \geqslant 0\right\}\right],
\end{aligned}
$$

where the min and max function return, respectively, the lowest and highest value of their arguments. In addition, it is useful to define for each phase point $x$ and each set of two nonoverlapping phase space regions $\left\{\Omega_{1}, \Omega_{2}\right\}$ the following characteristic functions:

$$
\begin{aligned}
& \bar{h}_{\Omega_{1}, \Omega_{2}}^{b}(x)= \begin{cases}1 & \text { if } h_{\Omega_{1}}\left(f\left(x,-t_{\Omega_{1} \cup \Omega_{2}}^{b}(x)\right)\right)=1, \\
0 & \text { otherwise, }\end{cases} \\
& \bar{h}_{\Omega_{1}, \Omega_{2}}^{f}(x)= \begin{cases}1 & \text { if } h_{\Omega_{1}}\left(f\left(x,+t_{\Omega_{1} \cup \Omega_{2}}^{f}(x)\right)\right)=1, \\
0 & \text { otherwise. }\end{cases}
\end{aligned}
$$


In other words, these functions measure whether a trajectory reaches $\Omega_{1}$ before $\Omega_{2}$ or not. As the system is ergodic, each phase space region will be visited in finite time and thus $\bar{h}_{\Omega_{1}, \Omega_{2}}^{b}(x)+\bar{h}_{\Omega_{2}, \Omega_{1}}^{b}(x)=\bar{h}_{\Omega_{1}, \Omega_{2}}^{f}(x)+\bar{h}_{\Omega_{2}, \Omega_{1}}^{f}(x)=1$ for any $x$. Using these definitions the characteristic functions for the overall states $\mathcal{A}$ and $\mathcal{B}$ are given by

$$
h_{\mathcal{A}}(x)=\bar{h}_{A, B}^{b}(x), \quad h_{\mathcal{B}}(x)=\bar{h}_{B, A}^{b}(x) .
$$

These states together span the complete phase space, as the system can never stay in the intermediate region between $A$ and $B$ forever. The overall states $\mathcal{A}$ and $\mathcal{B}$ do not sensitively depend on the definition of stable state $A$ and $B$ as long as it is reasonably. Of course, the stable regions should not overlap, and each trajectory between the stable states must be a true rare event for the reaction we are interested in. In addition, the probability that after this event the reverse reaction occurs shortly thereafter must be as unlikely as an entirely new event. In other words, the system must be committed to the stable states. Therefore, a reasonable definition of $A$ and $B$ requires that they should lie completely inside the basin of attraction of the respective two states $^{13}$ (see also Ref. 9). Special care has to be taken with this condition for processes which show many recrossings between state $A$ and $B$ before settling down. Such processes can occur in solution or in dilute gases. For instance, for organic reactions in aqueous solution, a rare specific hydrogen bonded network can lower the bond-breaking barrier and initiate the reaction. If the lifetime of those rare solvation structures is high, a sudden reverse reaction can occur as the barrier for the backward reaction is also lowered by the same amount. ${ }^{14-16} \mathrm{~A}$ similar phenomenon can happen in dilute gasses for which rare spontaneous fluctuations in the kinetic energy are the main driving force. A particle moving from one state to another due to a very high kinetic energy as result of sequence of collisions can cross the potential energy barrier several times before it will dissipate its energy by a new collision and relax into one of the stable states (see, e.g., Refs. 17, 18). These problems can in principle be solved by an adequate choice of the stable state definitions. For instance, the definition can depend explicitly on the presence of certain hydrogen bonds or on kinetic energy terms.

With our definition of overall states $\mathcal{A}$ and $\mathcal{B}$ we can write down our rate equation in the spirit of Eq. (4),

$$
k_{A B}=\frac{\left\langle h_{\mathcal{A}}\left(x_{0}\right) \dot{h}_{\mathcal{B}}\left(x_{0}\right)\right\rangle}{\left\langle h_{\mathcal{A}}\left(x_{0}\right)\right\rangle},
$$

where the dot denotes the time derivative taken at $t=0$. This rate expression does not depend on time although the evaluation of the characteristic functions still requires integration of the equations of motion. The transition from $\mathcal{A}$ into $\mathcal{B}$ takes place when the system coming from $A$ will cross the interface $\lambda_{B}$ for the first time (see Fig. 2). After this event the system will stay in $\mathcal{B}$. Equation (11) counts therefore only the first crossing through interface $\lambda_{B}$ and is hence equivalent to the effective positive flux expression,

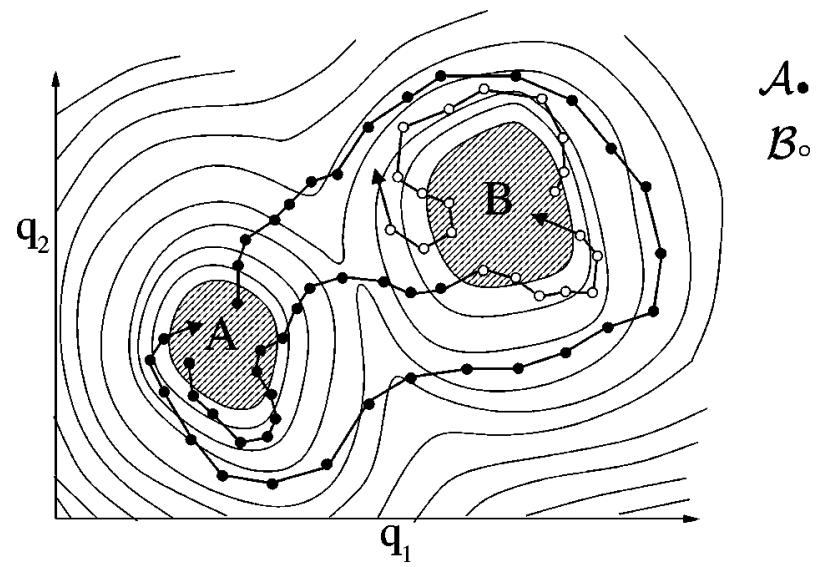

FIG. 2. Example of phase space regions in TIS. Thin solid curves denote the free energy contour lines. $q_{1}$ and $q_{2}$ are two arbitrary projections of the degrees of freedom. $A$ and $B$ are the two stable states. The dots on the three shown trajectories indicate the positions of the system at successive time steps. The overall state $\mathcal{A}$ and $\mathcal{B}$ are indicated by black and white dots, respectively. Only one trajectory starts in $A$ and ends in $B$ and is therefore a true reactive event. The system changes from state $\mathcal{A}$ into $\mathcal{B}$ when it enters region $B$ for the first time. It can leave stable region $B$ shortly thereafter, but never go back to $A$ in a short time. The stable regions have to be chosen to fulfill that condition.

$$
\begin{aligned}
k_{A B} & =\frac{\left\langle h_{\mathcal{A}}\left(x_{0}\right) \dot{\lambda}\left(x_{0}\right) \theta\left(\dot{\lambda}\left(x_{0}\right)\right) \delta\left(\lambda\left(x_{0}\right)-\lambda_{B}\right)\right\rangle}{\left\langle h_{\mathcal{A}}\left(x_{0}\right)\right\rangle} \\
& =\lim _{\Delta t \rightarrow 0} \frac{1}{\Delta t} \frac{\left\langle h_{\mathcal{A}}\left(x_{0}\right) \theta\left(\lambda_{B}-\lambda\left(x_{0}\right)\right) \theta\left(\lambda\left(x_{\Delta t}\right)-\lambda_{B}\right)\right\rangle}{\left\langle h_{\mathcal{A}}\left(x_{0}\right)\right\rangle} .
\end{aligned}
$$

Note the similarity with Eq. (1). Strictly speaking $\theta\left(\lambda_{B}\right.$ $\left.-\lambda\left(x_{0}\right)\right)$ is redundant in Eq. (12) as $h_{\mathcal{A}}\left(x_{0}\right)=0$ if $\theta\left(\lambda_{B}\right.$ $\left.-\lambda\left(x_{0}\right)\right) \neq 1$. The last expression in Eq. (12) is most suitable for a numerical approach with $\Delta t$ as the time step in a molecular dynamics simulation. Evaluation of Eq. (12) requires counting all phase space points which at $t=0$ are just about to cross interface $\lambda_{B}$ in one time step and will enter region $A$ before $B$ when integrating backward in time starting from $x_{0}$. Unfortunately, Eq. (12) is not very efficient from a computational point of view because only a very small fraction of phase points close to interface $\lambda_{B}$ actually belong to $\mathcal{A}$, leading to poor statistics. We can enhance the statistical accuracy by relating the flux through $\lambda_{B}$ to the flux through an interface closer to $A$. We therefore introduce a set of $n$ nonintersecting interfaces $\lambda_{1}, \lambda_{2}, \lambda_{3}, \ldots, \lambda_{n}$, each interface $\lambda_{i}$ closer to $A$ than the next interface $\lambda_{i+1}$ (see Fig. 3). We define the corresponding phase space regions $\Omega_{\lambda_{i}} \equiv\{x \mid \lambda(x)$ $\left.>\lambda_{i}\right\}$. In this way $\Omega_{\lambda_{B}}$ is equivalent to our stable state $B$, while $\Omega_{\lambda_{A}}$ is the phase space outside stable state $A$. By introducing the following definition:

$$
\begin{aligned}
\Phi_{A, \lambda_{i}}\left(x_{0}\right) \equiv & \lim _{\Delta t \rightarrow 0} \frac{1}{\Delta t} \bar{h}_{A, \Omega_{\lambda_{i}}}\left(x_{0}\right) \theta\left(\lambda_{i}-\lambda\left(x_{0}\right)\right) \\
& \left.\times \theta\left(\lambda\left(x_{\Delta t}\right)-\lambda_{i}\right)\right),
\end{aligned}
$$

Equation (12) reduces to 


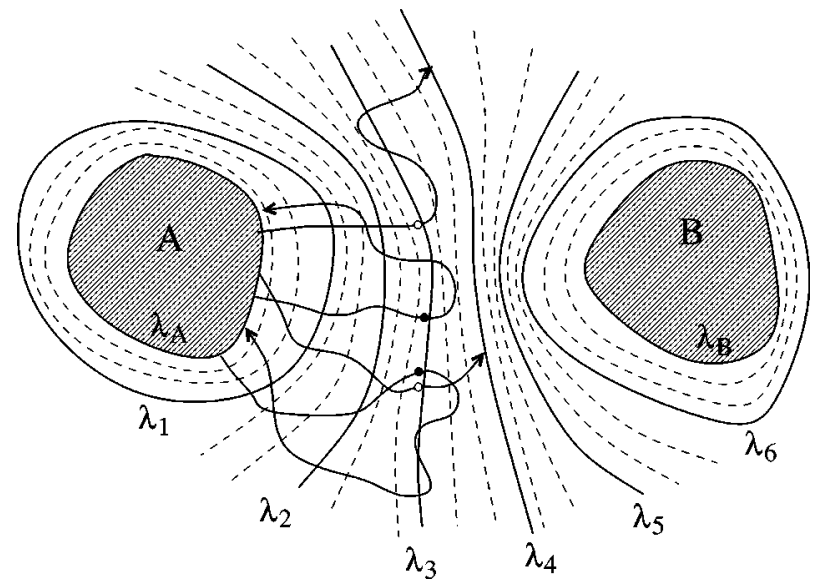

FIG. 3. Example of the division of the phase space by interfaces. $A$ and $B$ are the stable state regions with interfaces $\lambda_{A}$ and $\lambda_{B}$. The interfaces $\lambda_{1} \cdots \lambda_{6}$ correspond to a calculation of Eq. (16) with $n=6$. The dashed lines are the subinterfaces in between. Four trajectories are shown corresponding to a $\mathcal{P}\left(\lambda_{4} \mid \lambda_{3}\right)$ ensemble calculation. On each trajectory the $x_{0}$ time slice is indicated with a circle. Black circles correspond to $\bar{h}_{\Omega_{\lambda_{4}, A}}^{f}\left(x_{0}\right)=0$ and white circles correspond to $\bar{h}_{\Omega_{\lambda_{4}, A}}^{f}\left(x_{0}\right)=1$.

$$
k_{A B}=\left\langle\Phi_{A, \lambda_{B}}\right\rangle /\left\langle h_{\mathcal{A}}\right\rangle,
$$

where $\left\langle\Phi_{A, \lambda_{i}}\right\rangle$ denotes the effective positive flux through interface $\lambda_{i}$. The rate constant is thus equal to the effective positive flux through interface $\lambda_{B}$ with the condition the trajectories came directly from $A$. Note again that $\left\langle\Phi_{A, \lambda_{A}}\right\rangle /\left\langle h_{\mathcal{A}}\right\rangle$ is equal to the TST rate expression in Eq. (1) in case $\lambda^{*}=\lambda_{A}=\lambda_{B}$. The effective flux $\left\langle\Phi_{A, \lambda_{i}}\right\rangle$ can now be related to the effective flux $\left\langle\Phi_{A, \lambda_{i-1}}\right\rangle$ through an interface $\lambda_{i-1}$ closer to $A$ by (see Appendix)

$$
\left\langle\Phi_{A, \lambda_{i}}\left(x_{0}\right)\right\rangle=\left\langle\bar{h}_{\Omega_{\lambda_{i}}, A}^{f}\left(x_{0}\right)\right\rangle_{\Phi_{A, \lambda_{i-1}}} \times\left\langle\Phi_{A, \lambda_{i-1}}\left(x_{0}\right)\right\rangle,
$$

where $\langle\cdots\rangle_{\Phi_{A, \lambda_{i-1}}}$ denotes the ensemble average over all phase space points $x_{0}$ for which $\Phi_{A, \lambda_{i-1}}\left(x_{0}\right) \neq 0$. The factor $\left\langle\bar{h}_{\Omega_{\lambda_{i}}, A}^{f}\left(x_{0}\right)\right\rangle_{\Phi_{A, \lambda_{i-1}}} \equiv \mathcal{P}\left(\lambda_{i} \mid \lambda_{i-1}\right)$ is the conditional probability that a trajectory, coming from $A$, passes $\lambda_{i}$, given the fact that it has passed the interface $\lambda_{i-1}$ at an earlier time. By recursively substituting Eq. (15) into Eq. (14) the rate constant can be expressed as a product of conditional probabilities,

$$
\begin{aligned}
k_{A B} & =\frac{\left\langle\Phi_{A, \lambda_{1}}\right\rangle}{\left\langle h_{\mathcal{A}}\right\rangle} \prod_{i=1}^{n-1}\left\langle\bar{h}_{\Omega_{\lambda_{i+1}}^{f}, A}\right\rangle_{\Phi_{A, \lambda_{i}}}\left\langle\bar{h}_{B, A}^{f}\right\rangle_{\Phi_{A, \lambda_{n}}} \\
& \equiv \frac{\left\langle\Phi_{A, \lambda_{1}}\right\rangle}{\left\langle h_{\mathcal{A}}\right\rangle} \prod_{i=1}^{n-1} \mathcal{P}\left(\lambda_{i+1} \mid \lambda_{i}\right) \mathcal{P}\left(\lambda_{B} \mid \lambda_{n}\right) \\
& =\frac{\left\langle\Phi_{A, \lambda_{1}}\right\rangle}{\left\langle h_{\mathcal{A}}\right\rangle}\left\langle\bar{h}_{B, A}^{f}\right\rangle_{\Phi_{A, \lambda_{1}}} \equiv \frac{\left\langle\Phi_{A, \lambda_{1}}\right\rangle}{\left\langle h_{\mathcal{A}}\right\rangle} \mathcal{P}\left(\lambda_{B} \mid \lambda_{1}\right) .
\end{aligned}
$$

This expression is the central equation for TIS. Instead of just calculating the individual terms in the product of Eq. (16) we can equivalently determine a continuous crossing probability function $\mathcal{P}\left(\lambda \mid \lambda_{1}\right)$ for $\lambda$ between $\lambda_{1}$ and $\lambda_{B}$. This is reminiscent of umbrella sampling where a free energy dif- ference is usually estimated as a function of a continuous parameter $\lambda .{ }^{10}$ When calculating the ensemble average for $\mathcal{P}\left(\lambda_{i} \mid \lambda_{i-1}\right)$ we can also evaluate $\mathcal{P}\left(\lambda \mid \lambda_{i-1}\right)$ for interfaces $\lambda$ between $\lambda_{i-1}$ and $\lambda_{i}$ by dividing the phase space into a finer grid of sub interfaces (see Fig. 3). In this way we acquire useful information without significant extra cost, and, in addition, a measure for the convergence of the ensemble averages. The final monotonically decreasing crossing probability function $\mathcal{P}\left(\lambda \mid \lambda_{1}\right)$ can be obtained by matching the histograms from the different ensemble simulations. Techniques commonly applied in umbrella sampling such as overlapping windows between two successive ensemble averages and the use of biasing functions can also be employed here.

\section{THE TRANSITION INTERFACE SAMPLING ALGORITHM}

Inspection of Eq. (16) clearly shows that the TIS rate constant calculation is also a two step procedure. The first step, the effective flux $\left\langle\Phi_{A, \lambda_{1}}\right\rangle /\left\langle h_{\mathcal{A}}\right\rangle$ can be computed by simply running a MD simulation starting with a configuration in $A$ and counting the number of effective crossings. For an interface $\lambda_{1}$ close enough to stable state $A$ one can obtain a statistically accurate value.

The second part of the calculation consists of evaluating the product of the $\mathcal{P}\left(\lambda_{i+1} \mid \lambda_{i}\right)$ ensemble averages for the different interfaces $\lambda_{i}$ in Eq. (16). Here we need to sample all paths from region $A$ to either $A$ or $\Omega_{\lambda_{i+1}}$ that exhibit at least one crossing with interface $\lambda_{i}$. The Monte Carlo moves in TIS are very similar to the shooting move used in the TPS algorithm. The main difference is that the backward and forward integration is abandoned as soon as the edge of either $A$ or $\Omega_{\lambda_{i+1}}$ is reached. If the new path is accepted there is only one phase point $x$ along this path for which $\Phi_{A, \lambda_{i}}(x) \neq 0$, defining phase space point $x_{0}$. The shifting moves that were required in the original TPS implementation to enable proper sampling and improve statistical accuracy are here unnecessary.

To bootstrap the sampling procedure we first generate an initial path that starts in $A$, then crosses the interface $\lambda_{i}$ and finally ends in either $A$ or $\Omega_{\lambda_{i+1}}$ (see, for more details on initial path generation, Ref. 9). The phase space point $x_{0}$ is then defined as the first crossing point of this path with interface $\lambda_{i}$. Further, let $\tau=\operatorname{int}(t / \Delta t)$ be the discrete time slice index, and $\tau^{b} \equiv \operatorname{int}\left(t_{A}^{b}\left(x_{0}\right) / \Delta t\right)$ and $\tau^{f}$ $\equiv \operatorname{int}\left(t_{A \cup \Omega_{\lambda_{i+1}}}^{f}\left(x_{0}\right) / \Delta t\right)$ the forward and backward terminal time slice indices, respectively. Including $x_{0}$, the initial path then consists of $N^{(o)}=\tau^{b}+\tau^{f}+1$ time slices. With these definitions in mind is the TIS algorithm as follows:

(1) From the current path with length $N^{(o)}$ choose a random time slice $\tau$, with $-\tau^{b} \leqslant \tau \leqslant \tau^{f}$.

(2) Change all momenta of $x_{\tau \Delta t}$ by adding small random displacements $\delta p$ from a Gaussian distribution. Make sure the total momentum is conserved. ${ }^{9}$

(3) In case of a constant energy (NVE) simulation, rescale the new momenta to the old energy value and continue with step (4). In case of constant temperature (NVT) 
accept the new momenta (else reject the whole TIS move) with a probability ${ }^{10}$

$\min \left[1, \exp \left(\beta\left(E\left(x_{\tau \Delta t}^{(o)}\right)-E\left(x_{\tau \Delta t}^{(n)}\right)\right)\right)\right]$

Here, $E(x)$ is the total energy of the system at phase space point $x$.

(4) Integrate equations of motion backward in time by reversing the momenta at time slice $\tau$, until reaching either $A$ or $\Omega_{\lambda_{i+1}}$. Reject in the case of $\Omega_{\lambda_{i+1}}$ else continue with the next step.

(5) Integrate from time slice $\tau$ forward until reaching either $A$ or $\Omega_{\lambda_{i+1}}$. Reject if the entire trial path does not cross the interface $\lambda_{i}$, else continue with the next step.

(6) Accept the trial path with a probability

$\min \left[1, \frac{N^{(o)}}{N^{(n)}}\right]$,

where $N^{(n)}$ is the length of the new path. If accepted, replace the old path with the new one.

(7) Reassign $x_{0}$ to be the first crossing point with $\lambda_{i}$ and sample the value of $\bar{h}_{\Omega_{\lambda_{i+1}, A}^{f}}\left(x_{0}\right)$ to measure $\mathcal{P}\left(\lambda_{i+1} \mid \lambda_{i}\right)$.

(8) Repeat from step (1).

As usual in Monte Carlo schemes, any rejection along this route implies counting the old path again in the ensemble average. The acceptance probabilities at step (3) and step (6) are required to satisfy the detailed balance condition (see, e.g., Ref. 10).

Instead of generating a complete path and then accepting or rejecting accordingly to the probability at step (6), it is more efficient to determine a maximum path length in advance. Before embarking on the time consuming fourth and fifth step, we first take a uniform random number $\alpha$ between 0 and 1 and determine the maximum allowed path length by

$$
N_{\max }^{(n)}=\operatorname{int}\left(N^{(o)} / \alpha\right) .
$$

In this way we can directly stop the integration and reject the TIS move as soon the path length $N^{(n)}$ exceeds the maximum $N_{\max }^{(n)}$. In the course of the TIS simulation the pathlength fluctuates. This also means that the average path length becomes automatically shorter when changing from ensemble average $\mathcal{P}\left(\lambda_{i+1} \mid \lambda_{i}\right)$ to ensemble average $\mathcal{P}\left(\lambda_{i} \mid \lambda_{i-1}\right)$ closer to $A$.

The algorithm presented here does not require shifting moves because there is only one unique $x_{0}$ phase point along each pathway. However, one could consider the use of pathreversal moves as they have negligible computational cost and can sometimes facilitate ergodic sampling. ${ }^{9}$

\section{NUMERICAL RESULTS}

\section{A. The model}

We tested the TIS algorithm on a simple diatomic bistable molecule immersed in a fluid of purely repulsive particles. Such a system has previously been used in illustrating TPS rate constant calculations ${ }^{7}$ and is therefore a good starting point for a comparison between the two methods. The system consists of $N$ particles in two dimensions with interactions given by a pairwise Lennard-Jones (LJ) potential truncated and shifted at the minimum, often referred to as the Weeks-Chandler-Andersen (WCA) potential, ${ }^{19}$

$$
V_{\mathrm{WCA}}(r)=\left\{\begin{array}{l}
4 \epsilon\left[(r / \sigma)^{-12}-(r / \sigma)^{-6}\right]+\epsilon \text { if } r \leqslant r_{0} \\
0 \quad \text { if } r>r_{0},
\end{array}\right.
$$

where $r$ is the interatomic distance, and $r_{0} \equiv 2^{1 / 6} \sigma$. Throughout this section reduced units are used so that $\epsilon$ and $\sigma$, respectively, the LJ energy and length parameters, as well as the mass of the particles are equal to unity. The LJ unit of time $\left(m \sigma^{2} / \epsilon\right)^{1 / 2}$ is therefore also unity. In addition, two of the $N$ particles are interacting through a double well potential,

$$
V_{\mathrm{dw}}(r)=h\left[1-\frac{\left(r-r_{0}-w\right)^{2}}{w^{2}}\right]^{2} .
$$

This function has two minima separated by a barrier of height $h$ corresponding to the two stable states of the molecule: a compact state for $r=r_{0}$ and extended state for $r$ $=r_{0}+2 w$. For a high enough barrier, transitions between the states become rare and the rate constant is well defined. Hence, this system provides a useful test case for the TPS and TIS algorithms.

The system is simulated at a constant energy $E$ in a simulation square box with periodic boundary conditions. The total linear momentum is conserved and is set zero for all trajectories. The equations of motion are integrated using the velocity Verlet algorithm with a time step $\Delta t=0.002$. As in Ref. 7 we focus here on the computation of the rate constant for the isomerization reaction of the dimer from the compact state to the extended state. In the following section we describe general simulation details. In Sec. IV C we discuss the results for a system with a high enough barrier to avoid recrossings. Subsequently, we reproduce the simulations from Ref. 7 in Sec. IV D. These results do show recrossings, and we discuss the consequences for TPS and TIS.

\section{B. Methodology}

The TPS rate constant calculation evaluates the two factors in Eq. (6) separately as explained in Sec. II B. The first term in Eq. (6) is the ratio between the plateau value of the reactive flux correlation function $\left\langle\dot{h}_{B}(T)\right\rangle_{A, H_{B}(T)}$ and the correction $\left\langle h_{B}\left(t^{\prime}\right)\right\rangle_{A, H_{B}(T)}$. The second term $C\left(t^{\prime}\right)$ requires an umbrella sampling simulation in the form of a series of window calculations. An order parameter is chosen to define the characteristic functions of the stable states and to partition phase space in windows for the umbrella sampling. Besides shooting and shifting Monte Carlo moves to generate new paths in the transition path sampling we also employ a diffusion move that shifts the path by one time slice in arbitrary direction. This move is computationally very cheap but increases the statistics of the correlation functions. In all our simulations we therefore set the percentages for shooting, shifting and diffusion to $5 \%, 10 \%$, and $85 \%$, respectively. The parameters involved are always gaged such that the acceptance ratio is around $40 \%$ for shooting and shifting moves, ensuring an optimum efficiency of the sampling. ${ }^{7}$ 
The TIS method involves a direct determination of the flux and the calculation of the crossing probability functions $\mathcal{P}\left(\lambda_{i} \mid \lambda_{i-1}\right)$ between a series of successive interfaces as given by Eq. (16). The flux term in Eq. (16) is computed by means of a straightforward MD simulation starting in state $A$ and counting the number of effective positive crossings through interface $\lambda_{1}$, i.e., when the trajectory is directly coming from $A$. The second term in Eq. (16) is computed using the TIS algorithm of Sec. III. The basic requirement is a definition of a set of interfaces partitioning the phase space. Between these interfaces we defined a finer grid of subinterfaces to construct the crossing probability function $\mathcal{P}\left(\lambda \mid \lambda_{1}\right)$. As in the TPS calculation we adjusted the momentum displacement for the shooting move to give an acceptance of about $40 \%$.

Many parameters are involved in the two methods and to compare the relative efficiency we measured the CPU-time required for an arbitrary fixed error of $2.5 \%$ for each step in both the TPS and TIS calculations under the same computational conditions (1 GHz AMD Athlon). In both methods the final rate constant consists of a product of factors which have to be calculated independently. For each factor we performed $M$ simulation blocks of $N$ Monte Carlo cycles and adjusted $N$ such that after $M$ block averages the relative standard deviation of each term in Eqs. (4) and (11) was 2.5\%. The total CPU time is given by summing the individual $2.5 \%$ error CPU times for each factor. The final error in the rate constants is obtained by the standard propagation rules using all simulation results (i.e., not only the ones for the $2.5 \%$ error CPU time calculation).

\section{System with high energy barrier}

This system had a total number of particles $N=25$, and a total energy $E=25$. The square simulation box was adjusted to give a number density of 0.7 . The barrier height was $h=15$ and the width-parameter $w=0.5$, so that the minima of $V_{\mathrm{dw}}(r)$ were located at $r \simeq 1.12$ and $r \simeq 2.12$ while the top of the barrier was at $r \simeq 1.62$. In the TPS rate calculation we defined stable states $A$ and $B$ as $r<r_{A}=1.5$ and $r>r_{B}=1.74$, respectively. We computed the correlation function $\left\langle h_{B}(t)\right\rangle_{A, H_{B}(T)}$ using TPS with a fixed path length $T=2.0$. The correlation function is shown in Fig. 4 together with its time derivative, the reactive flux. The latter function clearly displays a plateau. Next, we chose four different $t^{\prime}$ $=0.1,0.3,1.0,2.0$ and performed umbrella sampling simulations using 8 windows to calculate $C\left(t^{\prime}\right)$. In each window we measured the probability to find the path's end point $r\left(t^{\prime}\right)$ at a certain value of $r$. These probability histograms were rematched and normalized. The final probability functions are shown in Fig. 5. Integration of the area under the histogram belonging to region B leads to $C\left(t^{\prime}\right)$ and finally to the rate constant. ${ }^{7}$ In Table I we give the values of the different contributions to the rate constant given by Eq. 6, together with the rate constant. We report the average relative computation time needed to reach the $2.5 \%$ error (see Sec. IV B) in Table II.

For the TIS calculations we use the same order parameter $r$ and the same definition for region $B$, i.e., interface $\lambda_{B}$

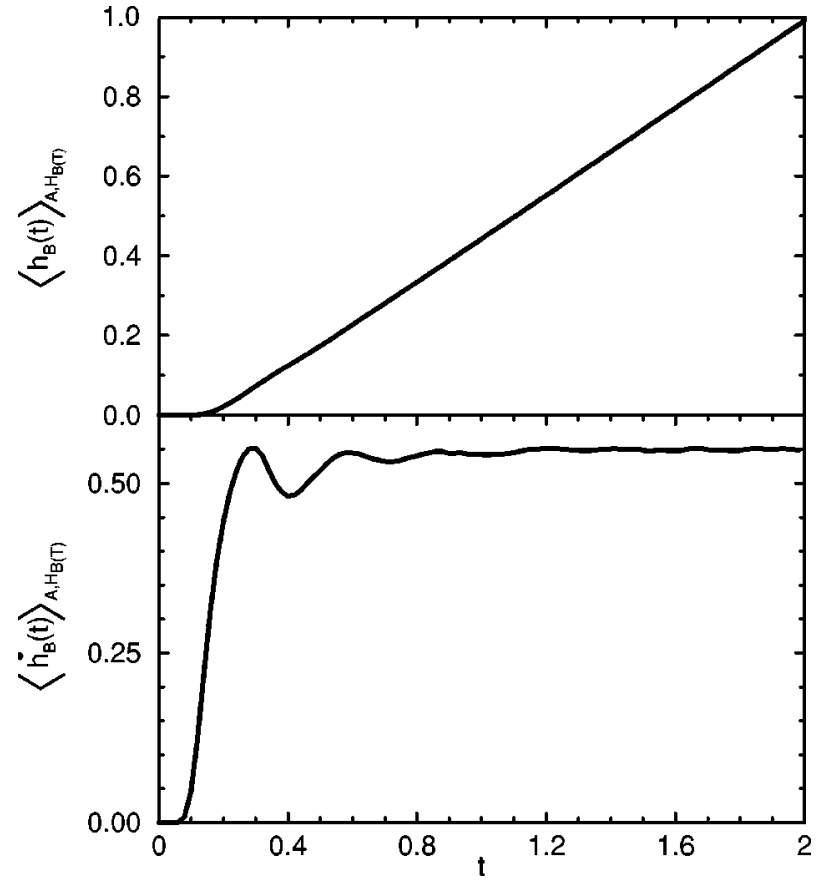

FIG. 4. TPS correlation function $\left\langle h_{B}(t)\right\rangle_{A, H_{B}(T)}$ (top) and its time derivative (bottom) for the system with high energy barrier. The error is comparable to line thickness.

is set at $r=1.74$. Stable state $A$ was defined by setting $\lambda_{A}$ $=\lambda_{1}$ at $r=1.24$. This interface is closer to the basin of attraction than the TPS stable state definition but yields a higher flux term $\left\langle\Phi_{A, \lambda_{1}}\right\rangle /\left\langle h_{\mathcal{A}}\right\rangle$ and gives better statistics. Note that the different definition of stable state $A$ does not change the final rate constant, as the overall state $\mathcal{A}$ does not sensitively depends on this definition. The flux term is calculated by straightforward NVE MD. As $\lambda_{A}$ is equal to $\lambda_{1}$ every positive crossing of this interface is counted in the flux

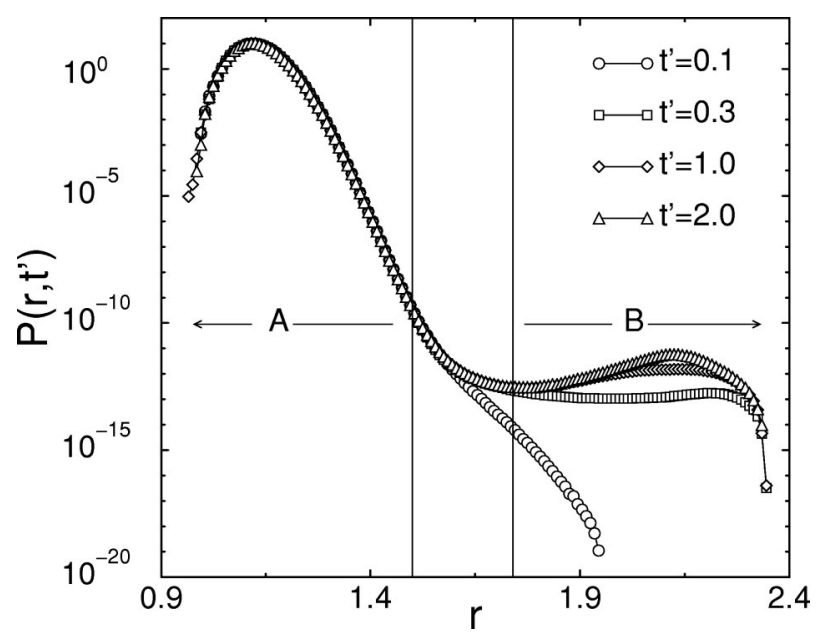

FIG. 5. TPS probability distributions $P\left(r, t^{\prime}\right)$ for four $t^{\prime}=0.1,0.3,1.0,2.0$ for the high energy barrier. The probability $P\left(r, t^{\prime}\right)$ is the chance that a path of length $t^{\prime}$ and starting in $A$ will have the end point conformation with a diatomic distance $r$. The graph is the result of the matching of eight window calculations. These eight windows are defined as $r<1.19,1.18<r<1.28$, $1.27<r<1.35, \quad 1.34<r<1.40, \quad 1.39<r<1.47, \quad 1.46<r<1.54, \quad 1.53<r$ $<1.75, r>1.74$. The errors on the histogram points are within the symbol size. 
TABLE I. Comparison of rate constants for the high energy barrier, computed with TPS at different $t^{\prime}$ and TIS. Contributing factors from Eq. (6) and Eq. (16) are also given.

\section{TPS}

\begin{tabular}{lccc} 
& $\left\langle\dot{h}_{B}(T)\right\rangle_{A B}$ & & \\
$t^{\prime}$ & $\left\langle h_{B}\left(t^{\prime}\right)\right\rangle_{A B}$ & $C\left(t^{\prime}\right) / 10^{-13}$ & $k_{A \rightarrow B} / 10^{-13}$ \\
\hline 0.1 & $3300 \pm 100$ & $0.0018 \pm 0.0001$ & $6.0 \pm 0.5$ \\
0.3 & $7.54 \pm 0.03$ & $0.76 \pm 0.02$ & $5.8 \pm 0.1$ \\
1.0 & $1.236 \pm 0.005$ & $4.8 \pm 0.3$ & $5.9 \pm 0.4$ \\
2.0 & $0.553 \pm 0.002$ & $11.4 \pm 0.9$ & $6.3 \pm 0.5$
\end{tabular}

TIS

\begin{tabular}{ccc}
$\left\langle\Phi_{A, \lambda_{1}}\right\rangle /\left\langle h_{\mathcal{A}}\right\rangle$ & $\mathcal{P}\left(\lambda_{B} \mid \lambda_{1}\right) / 10^{-13}$ & $k_{A \rightarrow B} / 10^{-13}$ \\
\hline $0.1196 \pm 0.0005$ & $49 \pm 1$ & $5.9 \pm 0.2$ \\
\hline \hline
\end{tabular}

because all trajectories must by default come directly from $A$. The conditional crossing probabilities $\mathcal{P}\left(\lambda_{i+1} \mid \lambda_{i}\right)$ in Eq. (16) are calculated for $n=5$ interfaces between the stable states (see Fig. 6). Between these interfaces we impose a finer grid to obtain the entire crossing probability function. The results for each stage and the final rate constant are shown in Table I. The rate constants of both methods agree within the statistical accuracy, showing that the TIS method is correct. In Table II we give the relative computation time to reach the $2.5 \%$ error for each term.

In comparing both methods we have to recall that the efficiency of TPS depends strongly on the choice of $t^{\prime}$. On the one hand the umbrella calculation of $C\left(t^{\prime}\right)$ is much faster for low values of $t^{\prime}$. But on the other hand the error in the correction term $\left\langle h_{B}\left(t^{\prime}\right)\right\rangle_{A, H_{B}(T)}$ increases for lower $t^{\prime}$. As a result there is an optimum $t^{\prime}$ for the error/CPU-time ratio, in this case approximately at $t^{\prime}=0.3$. Even for this optimized situation the TIS calculation is about two times faster. One could object that the correlation function in Fig. 4 has reached a plateau for $t=1.5$ already, reducing the TPS computation time by a factor $3 / 4$. But the choice for a path length $T=1.5$ cannot be taken without a priori knowledge. The first term in Eq. (6) implicitly depends on the path length $T$. Changing $T$ would alter the ensemble and might result in a different shape of the flux correlation function. We did not check this in detail, but we believe that $T$ cannot be chosen much smaller without introducing systematic errors. Further-

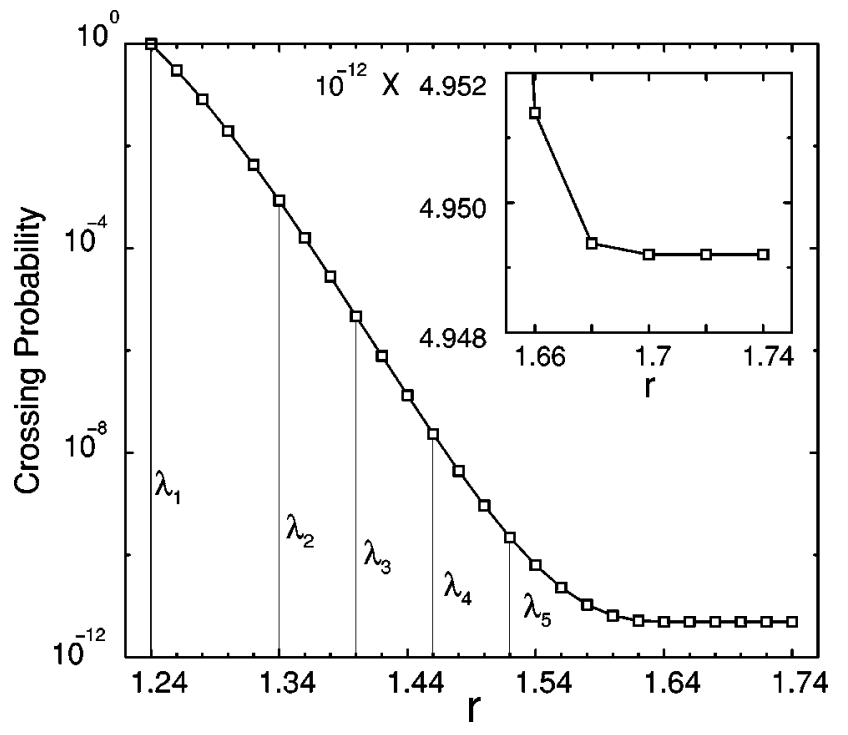

FIG. 6. TIS crossing probability $\mathcal{P}\left(\lambda \mid \lambda_{1}\right)=\left\langle\bar{h}_{\Omega_{\lambda}, A}^{f}\right\rangle_{\Phi_{A, \lambda_{1}}}$ as function of $\lambda$ $=r$ for the system with a high energy barrier. The function is computed by matching the five interface ensemble calculations. These interfaces were chosen at $\lambda_{1}=1.24, \lambda_{2}=1.34, \lambda_{3}=1.40, \lambda_{4}=1.46$, and $\lambda_{5}=1.52$. The error on the points is within symbol size. The inset is an enlargement in linear scale of the last part of the function. We clearly detect a horizontal plateau when approaching $\lambda_{B}$.

more, we emphasize here that we put much more effort in optimizing the TPS algorithm by tuning $t^{\prime}$, the windows, the ratio between shooting, shifting and diffusion moves than we did for TIS.

Figure 7 shows the histograms of path lengths for each TIS ensemble calculation and shows why TIS is faster. Sampling paths of fixed length with TPS results in spending unnecessary computation time inside the initial and final stable regions $A$ and $B$. In the TIS algorithm instead every path is adapted to its minimum length. Bringing the interface in closer to $A$ reduces these transition times. TIS optimizes itself during the simulation.

\section{System with low energy barrier}

In order to compare with previous results, we adopted the parameters from Ref. 7. The total number of particles

TABLE II. Comparison of CPU-times required for the $2.5 \%$ error at each stage for the system with the high energy barrier. The times are renormalized to the TIS total computation time. W1 to W8 denote the different windows used in the calculation, Int $\lambda_{1}$ to Int $\lambda_{5}$ denote the interface ensemble calculations.

TPS

\begin{tabular}{|c|c|c|c|c|c|c|c|c|c|c|}
\hline$t^{\prime}$ & $\frac{\left\langle\dot{h}_{B}(T)\right\rangle_{A B}}{\left\langle h_{B}\left(t^{\prime}\right)\right\rangle_{A B}}$ & W1 & W2 & W3 & W4 & W5 & W6 & W7 & W8 & Total \\
\hline 0.1 & 11.0 & 0.01 & 0.05 & 0.1 & 0.04 & 0.23 & 0.27 & 1.3 & 0.01 & 13.01 \\
\hline 0.3 & 0.2 & 0.01 & 0.14 & 0.28 & 0.13 & 0.58 & 0.43 & 0.19 & 0.02 & 1.98 \\
\hline 1.0 & 0.1 & 1.7 & 1.7 & 0.9 & 0.6 & 3.0 & 2.6 & 6.4 & 0.2 & 17.2 \\
\hline 2.0 & 0.1 & 0.03 & 1.8 & 4.5 & 4.4 & 15.3 & 8.0 & 20.3 & 0.6 & 55.03 \\
\hline
\end{tabular}

TIS

\begin{tabular}{lccccc}
$\left\langle\Phi_{A, \lambda_{1}}\right\rangle /\left\langle h_{\mathcal{A}}\right\rangle$ & Int $\lambda_{1}$ & Int $\lambda_{2}$ & Int $\lambda_{3}$ & Int $\lambda_{4}$ & Total time \\
\hline 0.07 & 0.265 & 0.09 & 0.15 & 0.21 & 0.215 \\
\hline \hline
\end{tabular}




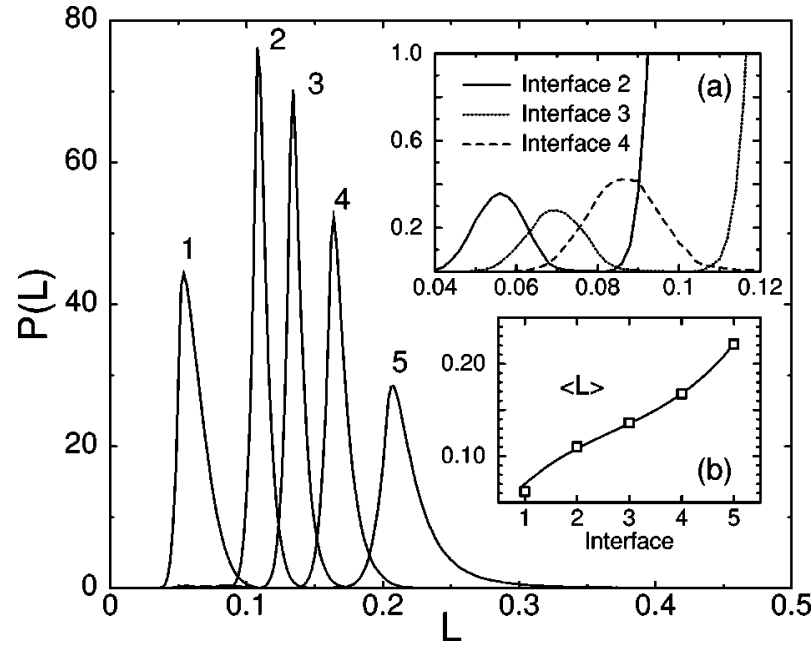

FIG. 7. Histograms $P(L)$ of path length $L$ for each ensemble, computed for the system with the high energy barrier. Inset (a) is an enlargement of the bottom left area, where windows 2, 3, 4 display a second peak. They represent that small fraction of paths that are able to cross all the interfaces up to the rightmost interface and do not have to return to A (cf. the trajectories with the white circle in Fig. 3). Inset (b): average path length in each window. At variance with TPS the TIS algorithm adapts the path length to the ensemble. In going from interface 5 to interface 1 one gets closer to state $A$ and the path length shortens accordingly.

was $N=9$, the total energy was $E=9$ and the square simulation box was adjusted for a number density of 0.6. The barrier height is $h=6$ and the width-parameter is $w=0.25$. Minima are at $r \simeq 1.12$ and $r \simeq 1.62$, while the top of the barrier is at $r \simeq 1.37$. This barrier is much lower than in the previous section resulting in more frequent transitions. An approximate rate constant could even be achieved by straightforward MD simulations.

For the TPS calculations we defined the stable states $A$ and $B$ by $r<r_{A}=1.30$ and $r>r_{B}=1.45$, respectively. ${ }^{7}$ Using standard TPS simulation we computed the correlation function $\left\langle h_{B}(t)\right\rangle_{A, H_{B}(T)}$ with a total path length $T=2$ (shown in Fig. 8). Next, we measured the probability histograms to find the paths end point at a certain order parameter value $r$ for four different times $t^{\prime}=0.1,0.4,0.8,2.0$, using five windows ${ }^{7}$ (see Fig. 9). As described in the previous section, matching the probability histograms and subsequent integration leads to $C\left(t^{\prime}\right)$. The resulting final rate constants, shown in Table III, are comparable with the results of Ref. 7, but more accurate. We will discuss these values after giving the results of the TIS.

Figure 10 shows that fast recrossings can occur for a low barrier, implying that $r$ alone is not sufficient as an order parameter to define the stable states in the simulations. Apparently, this does not effect the TPS results much, but it is very important for TIS because of the assumption that stable region B is really stable and recrossings do not take place. To ensure the stability of the TIS stable states we chose a new order parameter that not only depends on the interatomic distance $r$ in the dimer but also on a kinetic term, given by $\dot{r}$. The stable states can then be defined by

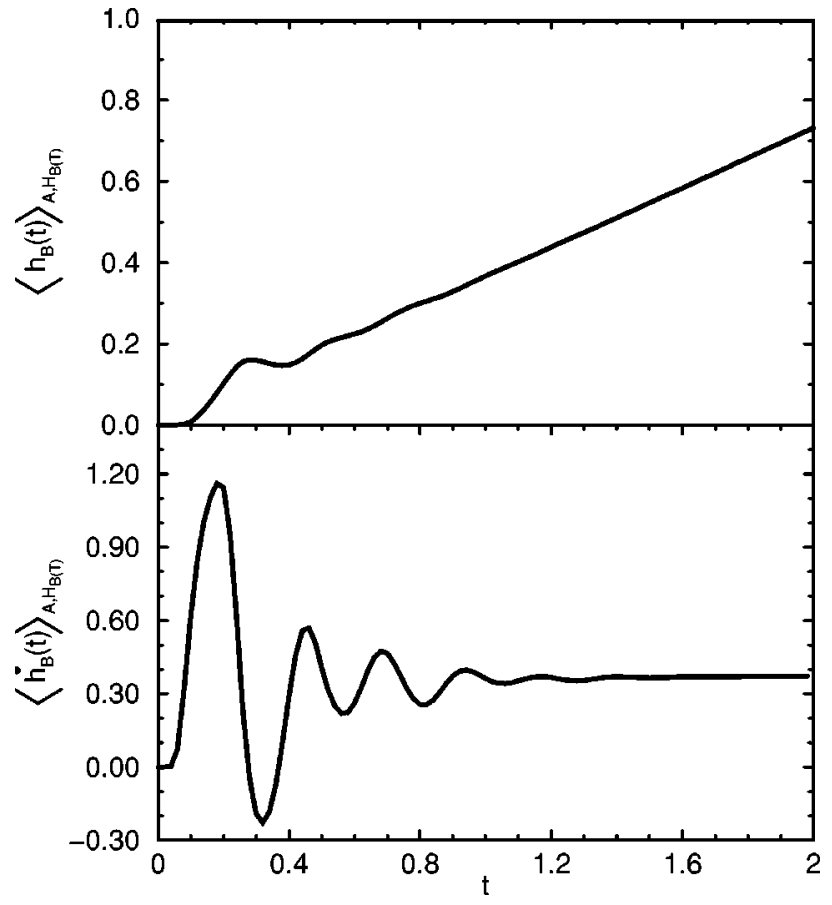

FIG. 8. TPS correlation function $\left\langle h_{B}(t)\right\rangle_{A, H_{B}(T)}$ (top) and its time derivative (bottom) for the system with low energy barrier. The error is comparable to line thickness.

$$
\begin{aligned}
& E_{d}(r, \dot{r}) \equiv \frac{\dot{r}}{4}+V_{\mathrm{dw}}(r), \\
& x \in A \text { if } r<1.37 \text { and } E_{d}(r, \dot{r}) \leqslant 1.5, \\
& x \in B \text { if } r>1.37 \text { and } E_{d}(r, \dot{r}) \leqslant 1.5,
\end{aligned}
$$

where $E_{d}$ is the sum of the kinetic and potential energy of the dimer that has a reduced mass of $1 / 2$. In the $\{r, \dot{r}\}$ plane these

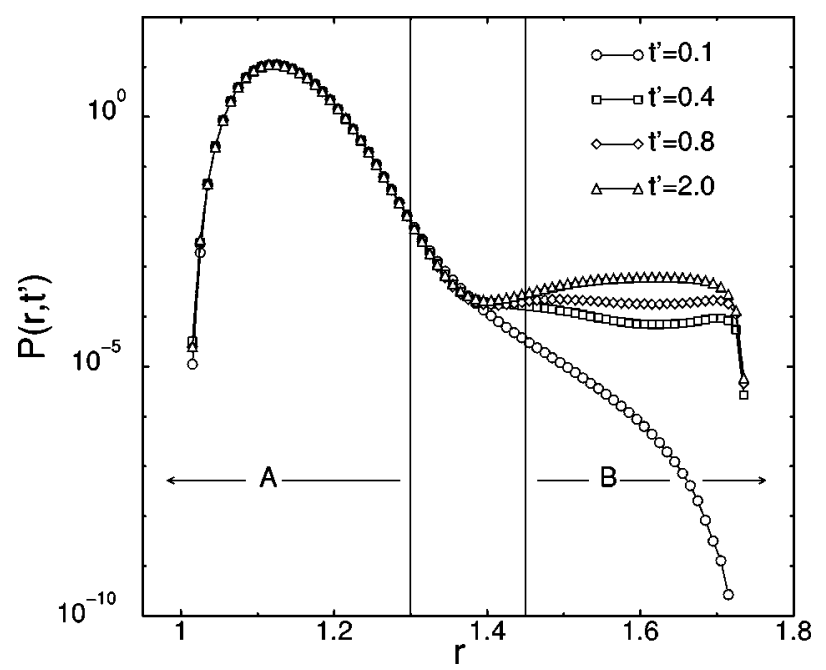

FIG. 9. TPS probability distributions $P\left(r, t^{\prime}\right)$ for four $t^{\prime}=0.1,0.4,0.8,2.0$ for the system with low energy barrier. $P\left(r, t^{\prime}\right)$ is defined as in Fig. 5. The graph is the result of the matching of five window calculations. These five window calculations are defined as $r<1.22,1.21<r<1.26,1.25<r$ $<1.30,1.29<r<1.46, r>1.45$. The errors on the histogram points are within the symbol size. 
TABLE III. Comparison of rate constants for the low energy barrier computed with TPS at different $t^{\prime}$ and with TIS, including the contributing factors from Eq. (6) and Eq. (16), respectively.

TPS

\begin{tabular}{lccc} 
& $\frac{\left\langle\dot{h}_{B}(T)\right\rangle_{A B}}{\left\langle h_{B}\left(t^{\prime}\right)\right\rangle_{A B}}$ & $C\left(t^{\prime}\right) / 10^{-5}$ & $k_{A \rightarrow B} / 10^{-5}$ \\
\hline$t^{\prime}$ & $47.3 \pm 0.2$ & $1.408 \pm 0.007$ & $6.67 \pm 0.04$ \\
0.1 & $2.505 \pm 0.007$ & $2.67 \pm 0.01$ & $6.68 \pm 0.03$ \\
0.4 & $1.240 \pm 0.003$ & $5.42 \pm 0.05$ & $6.72 \pm 0.07$ \\
0.8 & $0.507 \pm 0.001$ & $13.9 \pm 0.2$ & $7.03 \pm 0.09$ \\
2.0 & &
\end{tabular}

TIS

\begin{tabular}{ccc}
$\frac{\left\langle\Phi_{A, \lambda_{1}}\right\rangle}{\left\langle h_{\mathcal{A}}\right\rangle}$ & $\left\langle\bar{h}_{B, A}^{f}\right\rangle_{\Phi_{A, \lambda_{1}}} / 10^{-5}$ & $k_{A \rightarrow B} / 10^{-5}$ \\
\hline $0.2334 \pm 0.0003$ & $29.6 \pm 0.2$ & $6.90 \pm 0.06$ \\
\hline \hline
\end{tabular}

stable states form a D-shape and an inverse D-shape regions for $A$ and $B$, respectively (see Fig. 11). Crossing the interface $\lambda_{A}$ or $\lambda_{B}$ implies that the vibrational energy is decreased below the threshold, $E_{d}=1.5$. This threshold is made low enough to make fast recrossings to the other state unlikely. However, if we would have chosen it too low the paths would have become very long. We evaluated the crossing probability function in Eq. (16) for $n=3$ interfaces. The entire crossing probability function was obtained by partitioning the phase space in subinterfaces of the form $r=\lambda$ and $E_{d}(r, \dot{r})=\lambda$ as shown in Fig. 11. Note that in TIS multidimensional or multiple order parameters can be used in one simulation without a problem. This is more difficult in TPS, where a proper mapping of the complete phase space is required. Figure 12 shows the final rematched crossing probability. The monotonically decreasing function tends to reach a plateau on approaching the last interface. The last two values are not exactly equal but differ by $0.03 \%$, indicating that

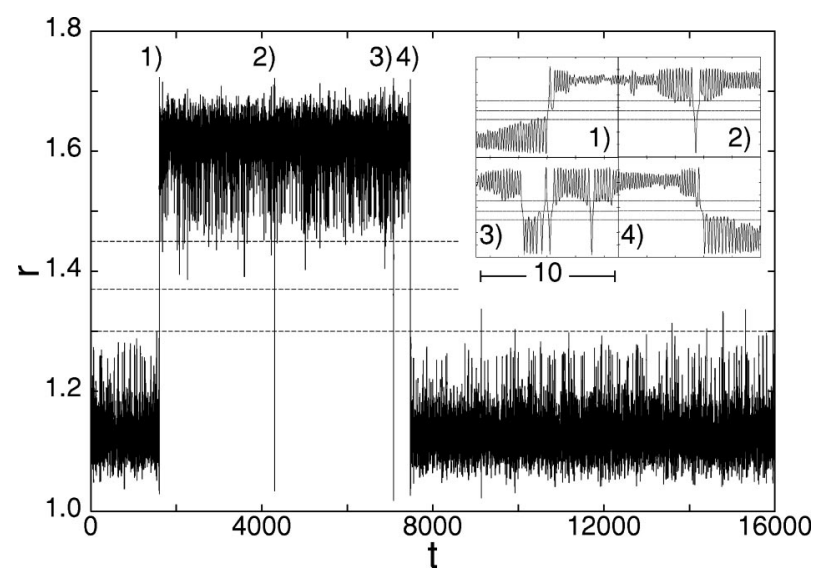

FIG. 10. Intramolecular distance of the dimer as function of time from a straightforward MD simulation for the system with the low energy barrier. Horizontal dashed line at 1.37 corresponds to the top of the potential barrier. Horizontal dashed lines at 1.3 and 1.45 correspond to the TPS state definitions of Ref. 7. Insets are enlargements of four typical events on a scale of 10. (1) and (4) correspond to true reactive events, $A \rightarrow B$ and $B \rightarrow A$, respectively, while (2) and (3) are nontrue, fast recrossing events. In particular, event (3) shows capricious behavior with many crossings of the barrier. The figure shows a clear separation of time scales, $t_{\mathrm{mol}} \sim 1$ and $t_{\mathrm{rxn}} \sim 1000$.

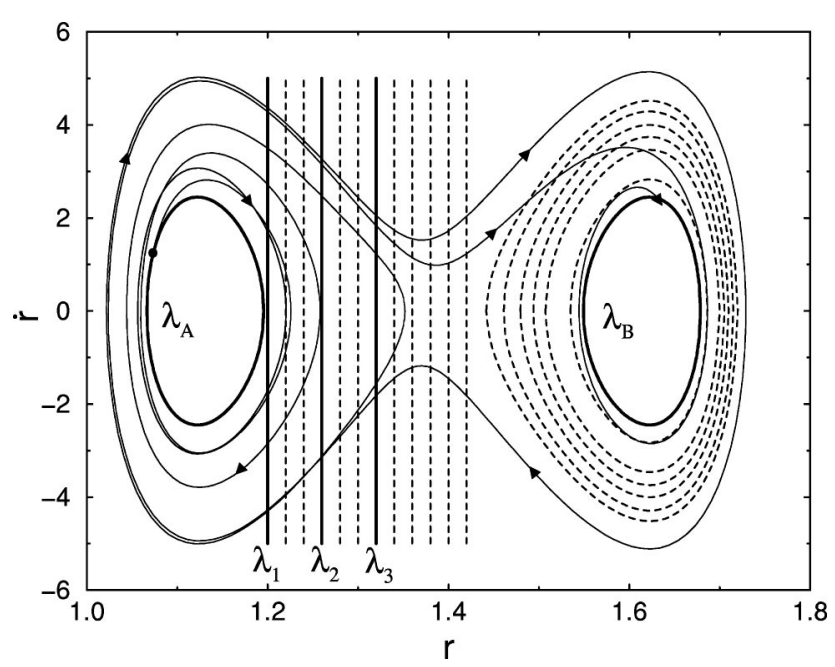

FIG. 11. One calculated path of the low energy barrier system shown in the $\{r, \dot{r}\}$ plane. The vertical solid lines are the interface $\lambda_{1}, \lambda_{2}$, and $\lambda_{3}$. The curves $\lambda_{A}$ and $\lambda_{B}$ are the boundaries of the TIS stable states. The dashed lines are the subinterfaces. The path starts at the dot on $\lambda_{A}$ and crosses the barrier three times before dissipating its energy and relaxing into state $B$.

a small fraction of the paths crossing the one but last subinterface still succeed to return to $A$ without crossing $\lambda_{B}$. This difference is comparable with the chance of a new independent transition (given by the rate constant). Note that without the kinetic energy definition for the stable states Eq. (20), the final crossing probability and thus the rate constant would have been overestimated by a factor $5 / 4$.

For the effective flux $\left\langle\Phi_{A, \lambda_{1}}\right\rangle /\left\langle h_{\mathcal{A}}\right\rangle$ calculation we performed MD simulations as described in Sec IV B. In contrast to the high barrier case, $\lambda_{1}$ is not equal $\lambda_{A}$, and not all positive crossings with $\lambda_{1}$ are effective crossings. We counted only the first crossing when the system left region $A$ and waited until the system fell back to region $A$ before

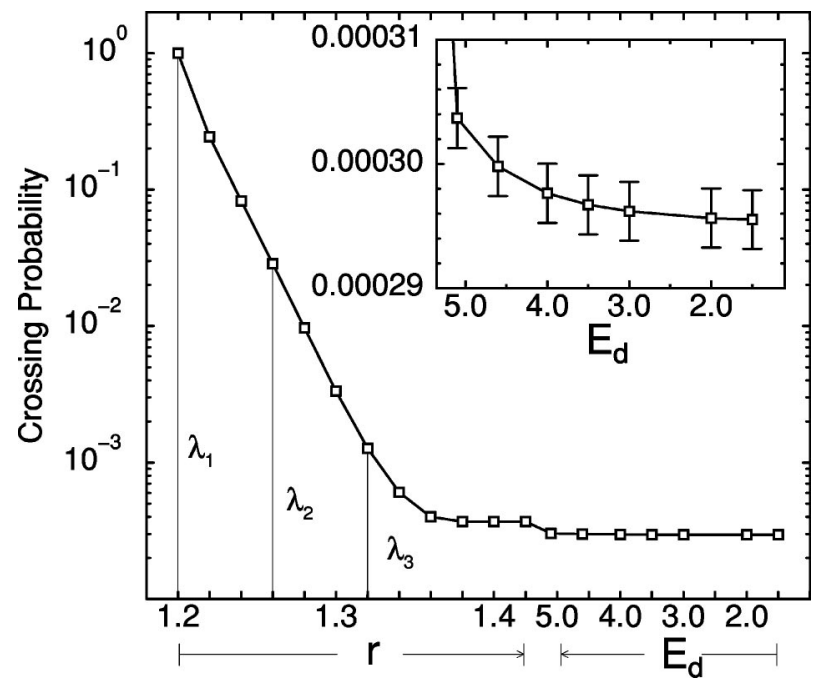

FIG. 12. The crossing probability $\mathcal{P}\left(\lambda \mid \lambda_{1}\right)$ for the system with the low energy barrier. The function is computed by matching ensemble calculations with interfaces $\lambda_{1}$ at $r=1.20, \lambda_{2}$ at $r=1.26$, and $\lambda_{3}$ at $r=1.32$. The inset is an enlargement of the final part. The function is converging to a plateau but has not yet reached it. The different values of the last points are due to the presence of fast recrossings. The error is inside the symbol size. 
TABLE IV. Comparison of CPU times required for the $2.5 \%$ error at each stage for the system with the low energy barrier. The times are renormalized to the TIS total computation time.

\begin{tabular}{|c|c|c|c|c|c|c|c|}
\hline \multicolumn{8}{|l|}{ TPS } \\
\hline \multirow[b]{2}{*}{$t^{\prime}$} & \multicolumn{7}{|l|}{$\left\langle\dot{h}_{B}(T)\right\rangle_{A B}$} \\
\hline & $\overline{\left\langle h_{B}\left(t^{\prime}\right)\right\rangle_{A B}}$ & W1 & W2 & W3 & W4 & W5 & Total \\
\hline 0.1 & 0.68 & 0.03 & 0.009 & 0.01 & 0.1 & 0.001 & 0.83 \\
\hline 0.4 & 0.4 & 0.09 & 0.03 & 0.04 & 0.25 & 0.01 & 0.82 \\
\hline 0.8 & 0.28 & 0.21 & 0.07 & 0.11 & 1.5 & 0.04 & 2.21 \\
\hline 2.0 & 0.35 & 0.28 & 0.38 & 0.93 & 7.27 & 0.14 & 9.35 \\
\hline \multicolumn{8}{|l|}{ TIS } \\
\hline$\left\langle\Phi_{A, \lambda_{1}}\right\rangle$ & \multicolumn{2}{|c|}{ Int $\lambda_{1}$} & \multicolumn{2}{|c|}{ Int $\lambda_{2}$} & \multicolumn{2}{|c|}{ Int $\lambda_{3}$} & Total \\
\hline \multicolumn{8}{|l|}{$\left\langle h_{\mathcal{A}}\right\rangle$} \\
\hline 0.015 & \multicolumn{2}{|c|}{0.085} & \multicolumn{2}{|c|}{0.45} & \multicolumn{2}{|c|}{0.45} & 1 \\
\hline
\end{tabular}

counting a new crossing. As the MD trajectory sometimes displayed a spontaneous transition to region $B$, we stopped the simulation and started again by replacing the system in a randomized configuration of $A$. Table III shows the final values and the corresponding errors of these calculations. The relative computation time for each term is detailed in Table IV.

If we compare the final results of Table III we see that the efficiency of TIS is more than nine times better than the TPS efficiency for $t^{\prime}=2$, and more than two times better than TPS value for $t^{\prime}=0.8$. But the TPS $t^{\prime}=0.1$ and $t^{\prime}$ $=0.4$ efficiencies are about $20 \%$ better than TIS. When we compare the rate constants, however, we notice that the TPS results for different $t^{\prime}$ do not agree. Among the TPS rate constants only the $t^{\prime}=2$ case is consistent with the TIS result. We believe that the $t^{\prime}=0.1$ and $t^{\prime}=0.4$ results suffer from systematic errors. For instance, for the shorter paths the TPS simulations might not be completely ergodic. Another explanation might be that a path length of $T=2$ is too short to allow convergence of the reactive flux. In the TIS calculation the average path length in the three interface simulations, from the closest to $B$ to the closest to $A$, is, respectively, 7.4, 4.3, and 0.63; much longer than the TPS path length (see Fig. 13). It is therefore surprising that the TPS approach with the simple stable state definition and very short paths still gives approximately the right rate constant. And indeed, when we computed the TPS correlation function with the TIS state definitions Eq. (20), we found that the path length had to be at least $T=20$ to see a plateau. We think that TPS works even with the simple state definitions and the short paths because both positive flux and negative flux terms contribute to Eq. (6). The TPS algorithm collects many paths of which some are not real transitions, but fast recrossings. The cancellation of positive and negative terms of these fast recrossing paths ensure the (almost) correct final outcome. In TIS each path must be true transition event and contributes as a positive term in rate Eq. (16), enhancing the convergence. This explains that the CPU time for the TIS calculation despite the much longer paths is still comparable with TPS one for low $t^{\prime}$. We note that the path ensemble using the more strict stable state definition is of course more useful in the analysis of the reaction mechanism.

For a more accurate comparison of the computation time we must keep the systematic errors lower than the statistical errors. In other words, we have to make sure that the results are converged. To test the convergence of the flux correlation function in TPS we can derive the following equality from Eq. (6),

$$
\frac{\left\langle h_{B}\left(t^{\prime}\right)\right\rangle_{A, H_{B}(T)}}{\left\langle h_{B}\left(t^{\prime \prime}\right)\right\rangle_{A, H_{B}(T)}}=\frac{C\left(t^{\prime}\right)}{C\left(t^{\prime \prime}\right)} .
$$

This equation is valid for any $t^{\prime}, t^{\prime \prime}<T$ if $T$ is large enough. We found that the equality does not hold for the system with the low barrier, indicating that $T$ is too low in the TPS calculation. Further examination of the flux correlation function $\left\langle h_{B}\left(t^{\prime}\right)\right\rangle_{A, H_{B}(T)}$ reveals that the apparent plateau has in fact a small positive slope. Calculations for higher values of $T$ suggest that one has to increase the path length at least to $T$ $=8$ to convergence to a plateau. With this in mind we think that the TIS computation is about a factor 5 more efficient than the TPS algorithm for the model system with the low barrier.

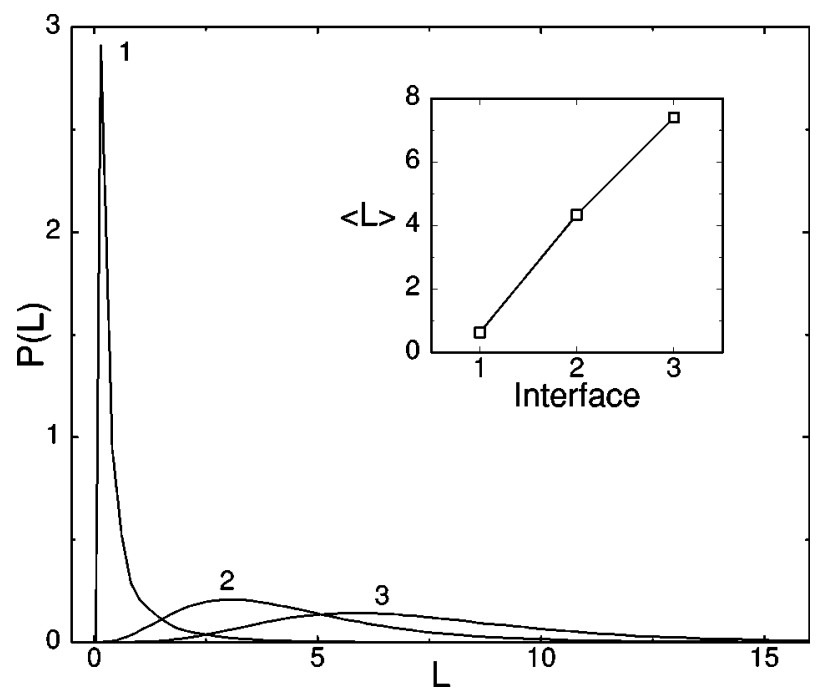

FIG. 13. Path length distribution $P(L)$ for each interface ensemble in the low energy barrier system. The inset shows the average path length in each ensemble. 


\section{CONCLUSION}

We developed a novel method, named transition interface sampling, for the calculation of rate constants based on transition path sampling concepts. Just as the original transition path sampling, the new method enables the calculation of rate constants of transitions between stable states separated by high free energy barriers without prior knowledge of the reaction coordinate. The new algorithm is different in spirit from the rate constant calculation that was introduced in Refs. 6, 7. In TPS the time correlation function $\left\langle h_{A}\left(x_{0}\right) h_{B}\left(x_{t}\right)\right\rangle /\left\langle h_{A}\right\rangle$ is determined for a single time using an umbrella sampling scheme followed by a calculation of the reactive flux prefactor in a separate path sampling simulation. The path length used in this simulation has to be long enough for the plateau to be reached. The TIS method advocated here calculates the rate constant by measuring the fluxes through a number of different interfaces and relating the flux through one interface to the next one. The big advantage of a flux instead of a correlation function is that trajectories going through the interfaces all contribute to the rate, whereas in TPS there are recrossings to be counted. In addition, the new method improves the original TPS method on a several other points. Once the interface is reached, the integration of motion can be stopped instead of going all the way to region $B$. In this way the TIS algorithm adapts itself to the optimal path length. One does not have to optimize the new method as much as TPS, where one has to find the optimal $t^{\prime}$ value and a proper balance between shooting and shifting. Besides being faster, the concept of calculating a flux comes natural with the rate constant definition, and implementation of the algorithm is hence simpler. Also, multidimensional or even discrete order parameters can easily be implemented in TIS. In the illustrative example we showed that we can obtain an increase in efficiency of at least a factor of 2-5 with respect to the TPS method used in Ref. 7 .

However, one has to be more careful in the definitions of the stable states, meaning that stable states have to be really stable. In TPS the choice of stable states is a bit more flexible as the final rate constant consists of cancellation of positive and negative terms. In Sec. IV D we showed how this problem for TIS can be solved by defining stable regions that explicitly depend on kinetic energy terms.

In summary, we believe that the TIS algorithm can make the rate constant calculation of many processes feasible that were hitherto difficult to obtain. For instance, chemical reactions in solution, isomerization of clusters, and conformational transitions in biomolecules. In a future publication we will report on these, more complex, applications.

\section{ACKNOWLEDGMENTS}

One of the authors (T.S.v.E) acknowledges NWO-CW (Nederlandse Organisatie voor Wetenschappelijk Onderzoek, Chemische Wetenschappen). P.G.B. acknowledges support from the FOM (Stichting Fundamenteel Onderzoek der Materie). We thank Evert Jan Meijer for carefully reading the manuscript.

\section{APPENDIX: FLUX RELATION}

In this Appendix we show how the effective flux $\Phi_{A, \lambda_{i}}$ can be related to the effective flux $\Phi_{A, \lambda_{i-1}}$ through an interface $\lambda_{i-1}$ closer to $A$. If at time $t=0$ a trajectory passes interface $\lambda_{i}$ while having started in $A$ some time earlier, there must always be a unique time when it passed interface $\lambda_{i-1}$ for the first time. Therefore we can write

$$
\Phi_{A, \lambda_{i}}\left(x_{0}\right)=\Phi_{A, \lambda_{i}}\left(x_{0}\right) \int_{0}^{t_{A \cup \Omega_{\lambda_{i}}}^{b}\left(x_{0}\right)} \mathrm{d} t \Phi_{A, \lambda_{i-1}}\left(x_{-t}\right)
$$

and hence,

$$
\begin{aligned}
& \left\langle\Phi_{A, \lambda_{i}}\left(x_{0}\right)\right\rangle \\
& =\int_{0}^{\infty} \mathrm{d} t\left\langle\Phi_{A, \lambda_{i-1}}\left(x_{-t}\right) \Phi_{A, \lambda_{i}}\left(x_{0}\right) \theta\left(t_{A \cup \Omega_{\lambda_{i}}}^{b}\left(x_{0}\right)-t\right)\right\rangle \\
& =\int_{0}^{\infty} \mathrm{d} t\left\langle\Phi_{A, \lambda_{i-1}}\left(x_{0}\right) \Phi_{A, \lambda_{i}}\left(x_{t}\right) \theta\left(t_{A \cup \Omega_{\lambda_{i}}}^{b}\left(x_{t}\right)-t\right)\right\rangle \\
& =\left\langle\Phi_{A, \lambda_{i-1}}\left(x_{0}\right) \int_{0}^{\infty} \mathrm{d} t \Phi_{A, \lambda_{i}}\left(x_{t}\right) \theta\left(t_{A \cup \Omega_{\lambda_{i}}}^{b}\left(x_{t}\right)-t\right)\right\rangle \\
& =\left\langle\Phi_{A, \lambda_{i-1}}\left(x_{0}\right) \int_{0}^{t_{A \cup \Omega_{\lambda_{i}}}\left(x_{0}\right)} \mathrm{d} t \Phi_{A, \lambda_{i}}\left(x_{t}\right)\right\rangle \\
& =\left\langle\Phi_{A, \lambda_{i-1}}\left(x_{0}\right) \bar{h}_{\Omega_{\lambda_{i}}^{f} A}\left(x_{0}\right)\right\rangle .
\end{aligned}
$$

The one, but last equation, follows because for each phase point $x$ and phase space region $\Omega$ it can be shown that $t$ $>t_{\Omega}^{f}(x) \Rightarrow t_{\Omega}^{b}(f(x, t)) \leqslant t \Rightarrow \theta\left(t_{\Omega}^{b}(f(x, t))-t\right)=0$. We rewrite the last expression of Eq. (A2) as a different ensemble average,

$$
\begin{aligned}
& \left\langle\Phi_{A, \lambda_{i-1}}\left(x_{0}\right) \bar{h}_{\Omega_{\lambda_{i}, A}^{f}}\left(x_{0}\right)\right\rangle \\
& =\frac{\left\langle\Phi_{A, \lambda_{i-1}}\left(x_{0}\right) \bar{h}_{\Omega_{\lambda_{i}}{ }^{f}}^{f}\left(x_{0}\right)\right\rangle}{\left\langle\Phi_{A, \lambda_{i-1}}\left(x_{0}\right)\right\rangle} \times\left\langle\Phi_{A, \lambda_{i-1}}\left(x_{0}\right)\right\rangle \\
& \equiv\left\langle\bar{h}_{\Omega_{\lambda_{i} A} A}^{f}\left(x_{0}\right)\right\rangle_{\Phi_{A, \lambda_{i-1}}} \times\left\langle\Phi_{A, \lambda_{i-1}}\left(x_{0}\right)\right\rangle,
\end{aligned}
$$

where $\langle\cdots\rangle_{\Phi_{A, \lambda_{i-1}}}$ denotes the ensemble average over all phase points $x_{0}$ for which $\Phi_{A, \lambda_{i-1}}\left(x_{0}\right) \neq 0$. The last equality gives rise to Eq. (15).

${ }^{1}$ J.C. Keck, Discuss. Faraday Soc. 33, 173 (1962); J.C. Keck, Adv. Chem. Phys. 13, 85 (1967).

${ }^{2}$ J.B. Anderson, J. Chem. Phys. 58, 4684 (1973).

${ }^{3}$ C.H. Bennett, in Algorithms for Chemical Computations, ACS Symposium Series No. 46, edited R.E. Christofferson (American Chemical Society, Washington, D.C., 1977), p. 63.

${ }^{4}$ D. Chandler, J. Chem. Phys. 68, 2959 (1978).

${ }^{5}$ C. Dellago, P.G. Bolhuis, F.S. Csajka, and D. Chandler, J. Chem. Phys. 108, 1964 (1998).

${ }^{6}$ P.G. Bolhuis, C. Dellago, and D. Chandler, Faraday Discuss. 110, 421 (1998).

${ }^{7}$ C. Dellago, P.G. Bolhuis, and D. Chandler, J. Chem. Phys. 110, 6617 (1999).

${ }^{8}$ P.G. Bolhuis, D. Chandler, C. Dellago, and P.L. Geissler, Annu. Rev. Phys. Chem. 53, 291 (2002), and references therein.

${ }^{9}$ C. Dellago, P.G. Bolhuis, and P.L. Geissler, Adv. Chem. Phys. 123, 1 (2002), and references therein. 
${ }^{10}$ D. Frenkel and B. Smit, Understanding Molecular Simulations, 2nd ed. (Academic, San Diego, 2002).

${ }^{11}$ D. Chandler, Introduction to Modern Statistical Mechanics (Oxford University Press, New York, 1987).

${ }^{12}$ Here, effective means that the recrossings through the interfaces are not being counted.

${ }^{13}$ In general, $A$ and $B$ are defined in phase space, but for most practical cases configuration space might be enough.
${ }^{14}$ M. Strnad, M.T.C. Martins-Costa, C. Millot, I. Tuñón, M.F. Ruiz-López, and J.L. Rivail, J. Chem. Phys. 106, 3643 (1997).

${ }^{15}$ Y. Okuno, J. Chem. Phys. 105, 5817 (1996).

${ }^{16}$ T.S. van Erp and E.J. Meijer (unpublished).

${ }^{17}$ Y.J. Cho, S.R. Van de Linde, L. Zhu, and W.L. Hase, J. Chem. Phys. 96, 8275 (1992)

${ }^{18}$ L. Sun, W.L. Hase, and K. Song, J. Am. Chem. Soc. 123, 5753 (2001).

${ }^{19}$ J.D. Weeks, D. Chandler, and H.C. Andersen, J. Chem. Phys. 54, 5237 (1971) 